\title{
Capacitance Mismatch Evaluation for Low-power Pipeline ADC Design
}

\author{
Tomohiko Ito, ${ }^{\text {a) }}$ Takafumi Yamaji, Daisuke Kurose, \\ and Tetsuro Itakura \\ Corporate Research and Development Center, Toshiba Corporation, \\ 1 Komukai Toshiba-cho, Saiwai-ku, Kawasaki, 212-8582, Japan \\ a)tomohiko2.ito@toshiba.co.jp
}

Abstract: It is known that the capacitance mismatch affects the nonlinearity of pipeline analog-to-digital converters (ADCs) and that larger capacitances tend to have a smaller mismatch. On the other hand, small capacitances are desirable because they reduce the current consumption. Therefore, to achieve a low-power, high-resolution $\mathrm{ADC}$, it is necessary to perform capacitance mismatch evaluation and determine the appropriate capacitance.

In this letter, a method for capacitance-mismatch measurement and the experimental results are described. The measurement results show that the mismatch is sufficiently small at the capacitance determined based on the noise requirement for 0.13- $\mu$ m CMOS ADCs.

Keywords: Analog-to-digital converter, ADC, capacitance mismatch Classification: Integrated circuits

\section{References}

[1] T. Ito, T. Yamaji, and T. Itakura, "A Relative Error Detection Circuit for MIM Capacitors," Proc. the 2003 IEICE Society Conf., Niigata, Japan, A-1-16, Sept. 2003. (in Japanese).

[2] S. H. Lewis, H. S. Fetterman, G. F. Gross, Jr., R. Ramachandran, and T. R. Viswanathan, "A 10-b 20-msample/s Analog-to-digital Converter," IEEE J. Solid-State Circuits, vol. 27, no. 3, pp. 351-358, March 1992.

[3] J. Hunter, P. Gudem, and S. Winters, "A Differential Floating Gate Capacitance Mismatch Measurement Technique," Proc. 2000 Intl. Conf. Microelectronic Test Structures (ICMTS 2000), pp. 142-147, March 2000.

[4] J. L. McCreary and D. A. Sealer, "Precision Capacitor Ratio Measurement Technique for Integrated Circuit Capacitor Array," IEEE Trans. Instrum. Meas., vol. IM-28, no. 1, March 1979.

[5] A. M. Abo and P. R. Gray, "A 1.5-V, 10-bit, 14.3-MS/s CMOS Pipeline Analog-to-digital Converter," IEEE J. Solid-State Circuits, vol. 34, no. 5, pp. 599-606, May 1999.

[6] K. Y. Kim, N. Kusayanagi, and A. A. Abidi, "A 10-b, 100-MS/s CMOS A/D Converter," IEEE J. Solid-State Circuits, vol. 32, no. 3, pp. 302-311, March 1997. 


\section{Introduction}

Pipeline architecture is often adopted for high-speed and medium-resolution analog-to-digital converters (ADCs). An advantage of pipeline ADCs is the large tolerance to the comparator offset. The dominant causes of the nonlinearity are the gain error of the residue amplifiers and the errors of the internal digital-to-analog converters (DACs) [2]. In many cases, a switchedcapacitor multiplying digital-to-analog converter (MDAC) is employed as the residue amplifier and the internal DAC. If the operational amplifier in the MDAC is assumed to be ideal, the error can be attributed to the mismatch between the capacitors in the MDAC.

One of the easy ways to reduce the mismatch is to make the capacitances large. For example, if $C_{U}$ is the unit capacitance and $\sigma_{u}$ is the standard deviation of the unit capacitances on a chip, the standard deviation of a capacitor that consists of two parallel unit-capacitors is $\sqrt{2} \sigma_{u}$, and the mismatch $\sqrt{2} \sigma_{u} / 2 C_{U}$ is smaller than $\sigma_{u} / C_{U}$.

However, a larger capacitance requires a larger drive current, and this results in larger power dissipation. Consequently, identification of the optimum capacitance value for MDACs is an important issue in low-power and high-resolution pipeline ADC design, and capacitance mismatch evaluation is indispensable.

In this letter, a capacitance mismatch evaluation method using a switched capacitor circuit and the experimental results are described.

\section{Mismatch detection circuit}

Our aim is to design a 10-bit or higher resolution ADC using CMOS technology, and the mismatch requirement is $2^{-10} \simeq 0.1 \%$ or smaller. In some reported 10-bit ADCs, $1 \mathrm{pF}$ or smaller capacitors are used [5, 6] and effective number of bits (ENOB) of more than 9.0 bits have been achieved. This means that a sufficiently small noise and sufficient linearity have been obtained with such small capacitors. To achieve a lower-power ADC, mismatch measurements at smaller capacitances are required.

At such small capacitances, however, accurate measurement is difficult because the parasitic capacitances on pads have similar values. Some techniques to measure capacitance mismatch have been reported $[3,4]$, but the techniques need precision measurement equipment.

Fig. 1 shows the proposed capacitance-mismatch detection circuit, which requires an ordinary clock source and an ordinary sampling oscilloscope, but no high precision equipment. The circuit consists of 4 capacitors to be tested, an operational amplifier with feedback capacitors, and switches.

To simplify the description, we make some assumptions. The validity of the assumptions is discussed in the next section. The assumptions are:

1. the offset of the amplifier is negligible,

2. the gain of the amplifier and the common mode rejection ratio (CMRR) are high enough, 


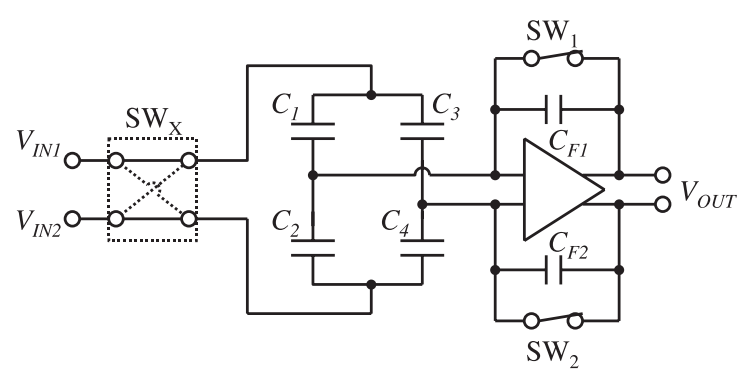

(a)

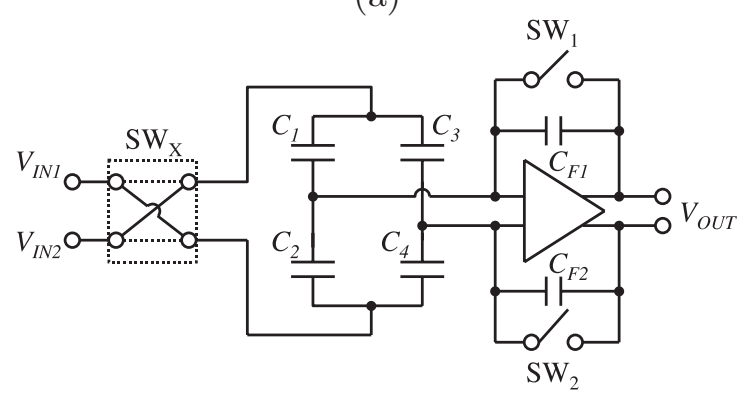

(b)

Fig. 1. Schematic of the proposed mismatch detection circuit: (a) in reset mode (b) in mismatch detection mode.

3. the average of $C_{1}, C_{2}, C_{3}$, and $C_{4}$ is $C_{0}$ and the standard deviations $\sigma_{i}$ for $\Delta C_{i}=C_{i}-C_{0}(i=1,2,3$, and 4$)$ are the same for all,

4. the feedback capacitors have no mismatch, i.e. $C_{F 1}=C_{F 2}=C_{F}$, and the deviation of the capacitance ratio $C_{F} / C_{0}$ is negligible,

5. all parasitic capacitances are negligible, and

6. the switches are ideal; i.e., there is no clock feed-through effect.

Under these assumptions, the circuit operation is considered to be composed of the following two steps.

First, the input switch $\mathrm{SW}_{X}$ connects the input and the capacitors directly as shown in Fig. 1 (a), and switches $\mathrm{SW}_{1}$ and $\mathrm{SW}_{2}$ short the input and output of the amplifier. We call this status 'reset mode'. The two input terminals of the amplifiers are virtually shorted, and the voltage at the input terminals is the same as the output common-mode voltage $V_{C O M}$. The capacitors have charges of $Q_{1 R}=C_{1}\left(V_{I N 1}-V_{C O M}\right), Q_{2 R}=C_{2}\left(V_{I N 2}-V_{C O M}\right)$, $Q_{3 R}=C_{3}\left(V_{I N 1}-V_{C O M}\right)$, and $Q_{4 R}=C_{4}\left(V_{I N 2}-V_{C O M}\right)$.

Next, as shown in Fig. 1 (b), the switches $\mathrm{SW}_{1}$ and $\mathrm{SW}_{2}$ are opened and the input switch $\mathrm{SW}_{X}$ turns to opposite status. The amplifier with feedback capacitors operates as a charge-to-voltage converter. We call this status 'mismatch detection mode'. The charges on the capacitors change to $Q_{1 D}=$ $C_{1}\left(V_{I N 2}-V_{C O M}\right), Q_{2 D}=C_{2}\left(V_{I N 1}-V_{C O M}\right), Q_{3 D}=C_{3}\left(V_{I N 2}-V_{C O M}\right)$, and $Q_{4 D}=C_{4}\left(V_{I N 1}-V_{C O M}\right)$, and the output voltage $V_{O U T}$ is

$$
V_{O U T}=\frac{\left(Q_{1 R}-Q_{1 D}\right)-\left(Q_{2 R}-Q_{2 D}\right)}{C_{F 1}}
$$




$$
\begin{aligned}
& -\frac{\left(Q_{3 R}-Q_{3 D}\right)-\left(Q_{4 R}-Q_{4 D}\right)}{C_{F 2}} \\
= & \frac{C_{1}-C_{2}-C_{3}+C_{4}}{C_{F}}\left(V_{I N 1}-V_{I N 2}\right) \\
= & \frac{\Delta C_{1}-\Delta C_{2}-\Delta C_{3}+\Delta C_{4}}{C_{F}} V_{I N},
\end{aligned}
$$

where $V_{I N}=V_{I N 1}-V_{I N 2}$. The output voltage depends on the capacitance mismatch. The detection circuit repeats these two steps and the output is observed with an oscilloscope. In our experiments, we used a sampling oscilloscope with an averaging function to reduce random noise.

From Eq. (1), the standard deviation of the output voltage $\sigma_{v}$ is

$$
\sigma_{v}=\frac{V_{I N}}{C_{F}} \sqrt{\sum_{i=1}^{4} \sigma_{i}^{2}}=\frac{2 V_{I N}}{C_{F}} \sigma_{1} .
$$

Therefore, the standard deviation of the capacitance mismatch is obtained to be

$$
\frac{\sigma_{i}}{C_{0}}=\frac{C_{F}}{2 C_{0}} \frac{\sigma_{v}}{V_{I N}}
$$

Consequently, the deviation of the capacitance mismatch is obtained by measuring the output voltages of the samples.

\section{Justification of the assumptions}

In this section, the validity of our assumptions is discussed. First of all, the allowable estimation error for the standard deviation should be clarified. We need only a rough estimate, and an error of $30 \%$ is acceptable. For example, when the capacitances are determined to make $3 \sigma$ within $0.5 \mathrm{LSB}, 99.7 \%$ of capacitor pairs have mismatches of less than 0.5 LSB. Even if the real $\sigma$ is $30 \%$ larger than estimated value, still $96.4 \%$ of capacitor pairs will have mismatches of less than 0.5 LSB. Therefore, an estimation error of $30 \%$ is acceptable.

Next, the nominal value in the mismatch detection circuit is discussed. The nominal value of $C_{F} / C_{0}$ is set as 0.5 , and $V_{I N}=V_{I N 1}-V_{I N 2}$ is set as $0.5 \mathrm{~V}$. According to Eq. (3), a $1-\mathrm{mV}$ deviation at the output corresponds to a capacitance mismatch of $0.05 \%$.

Under the above conditions, the following 6 effects are negligible.

1. Offset of the amplifier: The input offset of the amplifier is estimated to be a few $\mathrm{mV}$, and is detectable when the circuit is in the 'reset mode'. The offset effect is easily eliminated.

2. Gain and CMRR: The circuit simulation shows that the dc gain of the amplifier is more than $60 \mathrm{~dB}$ and CMRR is more than $80 \mathrm{~dB}$. Both figures are sufficiently high, because the error caused by the finite loop gain is less than $1 \%$ and the common-mode input signal is attenuated rather than amplified. 
3. Deviation of $C_{F} / C_{0}$ : The deviation is estimated to be less than a few $\%$, i.e. the deviation is much smaller than the nominal value. The deviation may result in a $\sigma_{v}$ error of a few $\%$. Note that the nominal values of $\Delta C_{i}(i=1,2,3,4)$ are 0 , and $\sigma_{i}$ is much larger than the nominal value. This difference makes $\sigma_{i}$ detectable, and makes the deviation of $C_{F} / C_{0}$ negligible.

4. Mismatch of the feedback capacitors: If $C_{F 1}=C_{F}(1+\alpha)$ and $C_{F 2}=$ $C_{F}(1-\alpha), \alpha$ is estimated to be less than a few $\%$. Equation (1) can be rewritten as

$$
\begin{aligned}
V_{O U T} & =\frac{Q_{12}}{C_{F 1}}-\frac{Q_{34}}{C_{F 2}} \\
& \simeq \frac{Q_{12}(1-\alpha)-Q_{34}(1+\alpha)}{C_{F}} \\
& =\frac{Q_{12}-Q_{34}-\alpha\left(Q_{12}+Q_{34}\right)}{C_{F}}
\end{aligned}
$$

where $Q_{12}=\left(Q_{1 R}-Q_{1 D}\right)-\left(Q_{2 R}-Q_{2 D}\right)$ and $Q_{34}=\left(Q_{3 R}-Q_{3 D}\right)-$ $\left(Q_{4 R}-Q_{4 D}\right)$. Equation (4) shows that $\alpha$ can affect the output voltage when $\left|Q_{12}-Q_{34}\right| \ll\left|Q_{12}+Q_{34}\right|$. However, the standard deviations of $Q_{12}-Q_{34}$ and $Q_{12}+Q_{34}$ should be the same, and $\alpha$ is much smaller than 1 . Thus the effect of $\alpha$ on the standard deviation is negligible.

5. Parasitic capacitances: The parasitic capacitances on the input nodes of the amplifier are negligible, because the nodes are virtually shorted. The capacitances on input switch $\mathrm{SW}_{X}$ and the output nodes may affect the frequency response of the circuit, but do not contribute to the output voltage.

6. Clock feed-through via $\mathrm{SW}_{X}$ : Even though the $\mathrm{SW}_{X}$, which consists of MOS transistors, injects a charge into the capacitors, the injected charge is absorbed by the low-impedance voltage source. As a result, the clock feed-through via the $\mathrm{SW}_{X}$ is negligible.

As for $\mathrm{SW}_{1}$ and $\mathrm{SW}_{2}$, the charge from the switches to the input nodes of the amplifier is considerable. When the injected charges are the same, the common-mode input voltage may change without affecting the differential output. When there is a mismatch, the output voltage changes.

To compensate the mismatch effect, two steps are added to the circuit operation and the four step operation is as follows:

In the first step, the circuit is set to reset-mode. The input switch $\mathrm{SW}_{X}$ connects the input and capacitors directly as shown in Fig. 1 (a), and switches $\mathrm{SW}_{1}$ and $\mathrm{SW}_{2}$ short the input and the output of the amplifier.

Next, switches $\mathrm{SW}_{1}$ and $\mathrm{SW}_{2}$ are opened, and the amplifier with feedback capacitors operates as charge-to-voltage converter. The switch $\mathrm{SW}_{X}$ remains connected as in reset mode. In this situation, the charge injection through $\mathrm{SW}_{1}$ and $\mathrm{SW}_{2}$ is converted to the output voltage. This mode is called the clock feed-through detection mode. 


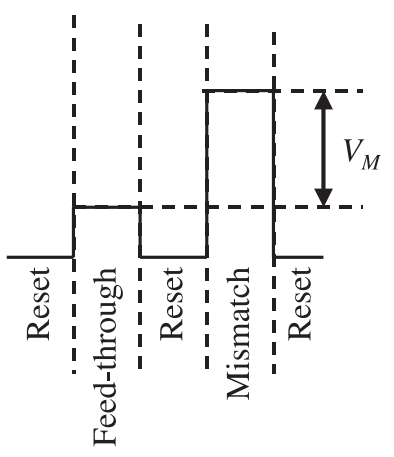

Fig. 2. Simplified output signal waveform

In the 3rd step, the circuit is set to reset mode again.

In the last step, the circuit is set to mismatch detection mode. In this mode, the switches $\mathrm{SW}_{1}$ and $\mathrm{SW}_{2}$ are opened, and the input switch $\mathrm{SW}_{X}$ is set to the opposite status as shown in Fig. 1 (b).

A diagram of the output signal waveform is shown in Fig. 2, and the desired output voltage $V_{M}$ is obtained as the difference between the voltage in the mismatch detection mode and that in the clock feed-through detection mode.

\section{Measured results}

The proposed circuit was fabricated using $0.13-\mu \mathrm{m}$ CMOS technology, and 32 samples were used. The capacitance for mismatch measurement was around $0.1 \mathrm{pF}$. The average was $0.4 \mathrm{mV}$ and the standard deviation was $0.8 \mathrm{mV}$. According to Eq. (3), a standard deviation of $0.8 \mathrm{mV}$ corresponds to a capacitance mismatch of $0.04 \%$.

\section{Conclusions}

The measured results indicate that the capacitance mismatch is not a serious problem for 10-bit ADC design because capacitances of which $3 \sigma$ is 0.5 LSB is $0.6 \mathrm{pF}$, and the capacitance of $0.6 \mathrm{pF}$ is smaller than capacitance to meet the noise requirements.

The trend toward deep submicron technology is reducing the power supply voltage, which in turn reducing the acceptable limits of the signal swing. This means that the permissible noise level is decreasing and a larger capacitance is required for the same resolution ADC. On the other hand, it is difficult to increase the capacitance per unit area, and the mismatch is not changing correspondingly. Therefore, at a 10 bit resolution, the capacitance mismatch is less important for low-power ADC design than thermal noise.

\section{Acknowledgement}

This work was supported by the Telecommunication Advancement Organization of Japan (TAO). 